\title{
Estudio observacional del contexto sociocognitivo de la catalogación de materias en bibliotecas universitarias
}

\author{
Um estudo de observação do contexto sociocognitivo \\ da catalogação de assunto em bibliotecas universitárias
}

An observation study of the socio-cognitive context of subject cataloguing in university libraries

\begin{abstract}
Vera Regina Casari Boccato (1), Mariângela Spotti Lopes FuJIta (2), Milena Polsinelli RUBI (3)
(1) Universidade Federal de São Carlos - UFSCar, campus de São Carlos, Departamento de Ciência de Informação, Rodovia Washington Luiz, Km 235, Campus Universitário, CEP 13565905 - São Carlos, SP - Brasil - Caixa Postal: 676, vboccato@ufscar.br. (2) Universidade Estadual Paulista Júlio de Mesquita Filho - UNESP, campus de Marília, Departamento de Ciência de Informação, Av. Hygino Muzzi Filho, 737, Campus Universitário, CEP 17525900 - Marília, SP - Brasil, Caixa Postal: 421, fujita@marilia.unesp.br. (3) Universidade Federal de São Carlos - UFSCar, campus de Sorocaba, Biblioteca, Rodovia João Leme dos Santos, Km 110 - SP-264, Bairro do Itinga, CEP 18052-780 - Sorocaba - SP - Brasil, milenarubi@ufscar.br
\end{abstract}

\section{Resumen}

Se presenta un estudio del proceso de catalogación temática en las bibliotecas universitarias desde la perspectiva socio-cognitiva en el que se analizan los procedimientos, los problemas y las percepciones de los bibliotecarios, usuarios y gestores. Se aplicaron protocolos verbales individuales y de grupo en bibliotecas universitarias. Se constató la ausencia de procedimientos sistemáticos para el análisis y la representación de los temas de los libros, la incompatibilidad del lenguaje documental, y la existencia de problemas de recuperación por materias en el catálogo. Se concluye que la tendencia actual de los catálogos es a funcionar como bases de datos. Por ello, el catalogador debe presentar una postura comprometida similar a la del indizador que trabaja en la producción de dichas bases.

Palabras clave: Indización. Catalogación. Catálogos colectivos. Bibliotecas universitárias. Perspectiva socio-cognitiva. Protocolo verbal.

\section{Introdução}

A biblioteca universitária está inserida em um contexto científico de alta especialização e, dessa maneira, organiza conhecimentos de várias áreas de assunto com o objetivo de contribuir com a universidade na promoção de um ensino de qualidade, voltado à formação de profissionais competentes para atuação em diversos setores.

Esse cenário é demonstrado pela aplicação das novas tecnologias de representação e recuperação da informação no contexto da organização do conhecimento que retrata uma mudança

\begin{abstract}
A study of the subject indexing process in university libraries is presented, using a socio-cognitive approach to analyze the procedures, difficulties and perceptions of the librarians, users, and managers that take part in the process. Individual and group verbal protocols were applied in the real environment of the cataloguers, the university libraries. The results were the absence of systematic procedures for book subject analysis and representation, the incompatibility of the indexing language, and problems in subject retrieval from the catalog. It is concluded that there is a tendency in catalogs to act as databases. Therefore, the cataloguer should present a level of commitment in his task similar to that of an indexer who works in the production of bibliographic databases.
\end{abstract}

Keywords: Indexing. Cataloging. Collective catalogs. University libraries. Socio-cognitive approach. Verbal protocol.

na postura de atuação do bibliotecário compromissado com a construção de catálogos coletivos online. Esse profissional assume uma responsabilidade não só com a sua comunidade usuária local (interna ou externa), mas também com uma comunidade usuária potencial remota que se torna cada vez mais exigente com a qualidade da informação disponibilizada pelos sistemas de recuperação da informação. Sendo assim, esses profissionais devem ser valorizados em suas funções, considerando-se os conhecimentos prévios, as visões de mundo, os conceitos e linguagens adquiridos durante seu processo de inter-relação com o meio social, 
como fatores contribuintes para a formação e desempenho satisfatório de um sistema de recuperação da informação.

No âmbito dos processos documentários o tratamento da informação envolve o conhecimento teórico e o metodológico disponível tanto para o tratamento descritivo do suporte material da informação quanto para tratamento temático de conteúdo da informação. No tratamento temático da informação, a indexação desempenha importante função ao possibilitar a análise de assunto para a representação do conteúdo documentário e, consequentemente, a recuperação deste em catálogos ou bases de dados. Esses dois canais de mediação entre usuário/pesquisador e documento refletem os processos de catalogação e indexação que estão envolvidos em divergências teóricas ligadas à história e evolução de cada um, ao ambiente onde são realizados, aos produtos gerados e aos profissionais que os executam.

A catalogação cooperativa para construção de catálogos trouxe um importante avanço com transferência e cópia de registros catalográficos, mas o processo de análise de assunto foi simplificado e, na maioria das vezes, foi eliminado. A análise de assunto, enquanto operação de tratamento de conteúdo documentário, é realizada, por exemplo, por indexadores e catalogadores com diferentes tipos de documentos e em diferentes sistemas de informação; no entanto, existe a possibilidade do catalogador não ter a compreensão de que realiza a indexação quando estabelece termos para os documentos no campo de Assunto Tópico de formatos bibliográficos, a exemplo o MARC 21.

Diante do exposto, nosso objetivo é avaliar o processo de indexação na catalogação para a construção de catálogos coletivos de bibliotecas universitárias. Para tanto, a perspectiva sociocognitiva será utilizada para análise de procedimentos, dificuldades e percepções de bibliotecários, usuários e dirigentes durante o processo de indexação.

\section{Indexação e catalogação de assunto: traços distintos entre dimensões teórico- metodológicas}

O tratamento da informação realizado pelo catalogador atua em dois níveis na organização e representação do conhecimento: da descrição bibliográfica referente ao tratamento físico da informação, centrados no suporte/documento e da descrição temática da informação, voltada para a representação e condensação do assunto intrínseco ou extrínseco tratado em um determinado documento (Guimarães, 2003).
Por sua vez, o tratamento temático, em bibliotecas, diz respeito ao assunto tratado no documento, ou seja, compreende a análise documentária como área teórica e metodológica que abrange as atividades de classificação, elaboração de resumos, indexação e catalogação de assunto, considerando as diferentes finalidades de recuperação da informação.

De acordo com nossa proposta de estudo, torna-se fundamental discutirmos os aspectos teóricos envolvendo as divergências entre os termos catalogação de assunto e indexação.

O termo "catalogação de assunto" (subject cataloguing) apresenta influência norte-americana e remonta a Charles Ammi Cutter que, em 1876, apresenta sua obra Rules for a dictionary catalog, com o objetivo de estabelecer regras para a formação de cabeçalhos alfabéticos de assuntos, que formariam catálogos alfabéticos de assunto.

Para Cutter (1904), os objetivos da catalogação de assunto eram: permitir ao usuário do catálogo encontrar um documento particular do qual o assunto é conhecido; encontrar outros documentos sobre o mesmo assunto ou sobre assuntos relacionados; dar assistência ao usuário na seleção de registros recuperados, o documento mais adequado às suas necessidades informacionais.

Fiúza (1985, p. 257) define a catalogação de assunto como "[...] a disciplina ou conjunto de disciplinas que tratam da representação, nos catálogos de bibliotecas, dos assuntos contidos no acervo."

A catalogação de assunto, termo adotado pela Declaração de Princípios Internacionais de Catalogação, realizada em 2003 na cidade de Frankfurt, compreende "A parte do processamento bibliográfico que fornece cabeçaIhos/termos de assunto ou classificações" (Tillett, 2009, p. 38). Essa terminologia é utilizada nos Estados Unidos, bem como na Austrália, Nova Zelândia, Canadá e República Tcheca para orientações ao uso da lista de cabeçalhos de assunto da Library of Congress Subject Headings (LCSH) da Library of Congress (Estados Unidos), no desenvolvimento de atividades de análise e representação temática da informação.

A indexação (indexing), que se origina da corrente teórica inglesa, é de acordo com os "Princípios de indexação" do World Scientific Information Programme (UNISIST 1981, p. 84) a indexação é "[...] a ação de descrever e identificar um documento de acordo com seu assunto." (Rubi, 2008, p. 24-25). 
Pinto Molina (1993, p. 208) relata que a indexação

[...] é a técnica de caracterizar o conteúdo de um documento [...] retendo as idéias mais representativas para vinculá-las a termos de indexação adequados.

A indexação é vista por Van Slype (1991) como a operação que consiste em enumerar os conceitos sobre os quais trata um documento e representá-los por meio de uma linguagem combinatória, lista de descritores livres, lista de autoridades e os tesauros de descritores - tendo como finalidade a busca documentária que será realizada a partir dos índices ou dos catálogos.

Nessa conceituação, observamos que o referido autor (Van Slype, 1991) enfoca a representação dos conceitos, por meio de uma linguagem específica com vistas ao processo de recuperação da informação por meio de índices ou catálogos, ratificando a nossa opinião sobre a realização da indexação na catalogação no processo de construção de catálogos de assunto.

O processo de indexação é composto por diferentes etapas, sobre as quais Lancaster (2004) e Pinto Molina (1993) afirmam que não precisam necessariamente ser realizadas de maneira seqüencial, uma vez que o indexador profissional já familiarizado com o processo pode realizá-las simultaneamente.

Com base nessas considerações, observamos que as divergências entre a catalogação de assunto e a indexação tornam-se mais evidentes quando autores como Lancaster (2004) e Silva e Fujita (2004) reconhecem a indexação e a catalogação de assuntos como conceitualmente equivalentes.

Lancaster (2004, p. 15-16) explica que a diferença presente na literatura da área sobre as expressões catalogação de assuntos e, indexação são inexpressivas e causadoras de confusão.

Para o referido autor (Lancaster, 2004, p. 15-16) o termo catalogação de assunto tem influência norte-americana e seu uso foi reforçado com a utilização das listas de cabeçalhos de assunto "[...] para representar o conteúdo total de itens bibliográficos completos (livros, relatórios, periódicos, etc.) [...]" e teve sua origem ligada principalmente à construção de catálogos de assuntos de bibliotecas nas quais são determinados os cabeçalhos de assunto.

Sobre a indexação, o autor (Lancaster, 2004, p. 15-16) relata ser "

[...] uma expressão utilizada de modo mais impreciso; refere-se à representação do conteúdo temá- tico de partes de itens bibliográficos completos, como é o caso de um índice no final de um livro".

Silva e Fujita (2004, p. 142) ressaltam semeIhanças e diferenças entre indexação alfabética de assunto e catalogação de assunto.

A indexação alfabética de assunto está vinculada à determinação de cabeçalhos de assuntos e por isso é, em alguns casos, também denominada de catalogação de assuntos. Apesar das divergências sobre semelhanças e diferenças entre os termos, a indexação alfabética de assuntos e a catalogação de assuntos são equivalentes porque são resultados de um mesmo processo: a análise de assunto.

Além disso, as referidas autoras Silva e Fujita (2004, p. 142) afirmam que a distinção entre os dois processos está na utilização de diferentes linguagens documentárias (lista de cabeçalho de assunto para catalogação de assunto e tesauros para indexação) e nos resultados dos dois processos que terão como produto final o índice e o catálogo de assunto.

Observamos, portanto, que os traços distintos que identificam as diferenças teóricas e metodológicas entre a catalogação de assunto e a indexação variam em função do contexto de atuação profissional, dos tipos de suportes em análise e da profundidade com que os processos são efetuados.

Neste momento, ressaltamos, também, dois aspectos que consideramos importante para a compreensão e diferenciação dos processos de catalogação de assunto e indexação:

1. ambos derivam de processos intelectuais para determinação de assuntos que melhor representem o documento para sua posterior recuperação, seja por meio de índices ou por meio de catálogos; e

2. a tendência atual dos catálogos em atuarem como bases de dados, inclusive com disponibilização de textos completos.

Apesar das considerações sobre os elementos que diferenciam a catalogação de assunto e a indexação apresentadas até o momento, entendemos que a indexação é o processo que, conforme perspectiva teórica e prática relatada até aqui, apresenta melhor sistematização de procedimentos e avaliação de desempenho na recuperação da informação que são vantagens importantes a serem acrescentadas na evolução atual dos catálogos online.

Isso posto, e uma vez que esta pesquisa aborda o processo de indexação na catalogação, é conveniente esclarecer que o catalogador ao realizar a indexação na catalogação, deverá ser entendido como indexador, pois verificamos que 
a própria área de Organização e Representação do Conhecimento reconhece a indexação e a catalogação de assuntos como processos conceitualmente equivalentes nas concepções de Lancaster (2004), Silva e Fujita (2004), conforme apresentações realizadas.

Todavia, não podemos deixar de mencionar a importância de aspectos que norteiam o "fazer" do bibliotecário no contexto da indexação que versam sobre política, o manual e a qualidade da indexação.

Sobre a política de indexação do sistema, Carneiro (1985, p. 221) expõe que

[...] deve servir como um guia para tomada de decisões, deve levar em conta os seguintes fatores: características e objetivos da organização, determinantes do tipo de serviço a ser oferecido; identificação dos usuários, para atendimento de suas necessidades de informação e recursos humanos, materiais e financeiros, que delimitam o funcionamento de um sistema de recuperação de informações.

Para Rubi (2008, p. 50)

[...] a política de indexação não deve ser vista como uma lista de procedimentos a serem seguidos, e sim uma filosofia que reflete os interesses e objetivos da biblioteca.

Entendemos, dessa maneira, que a qualidade da indexação está intimamente relacionada com uma política de indexação bem definida, contemplando aspectos relativos a:

1. consistência na especificidade dos termos atribuídos a um documento e no nível de exaustividade atingido na indexação;

2. qualificações do indexador (imparcialidade, conhecimento, etc.);

3. qualidade dos instrumentos de indexação (Associação Brasileira de Normas Técnicas, (1992, p. 3-4).

Dessa maneira, e subsidiado por Boccato (2009, p. 71-72), salientamos que o processo de indexação para catalogação é de responsabilidade de cada bibliotecário indexador, voltado para a realização de uma representação temática condizente com os conteúdos dos documentos (expressão do autor) e das necessidades informacionais de sua demanda, isto é, do usuário do seu sistema de recuperação da informação, exemplificado pelos catálogos coletivos online.

\section{Metodologia}

Em Ciência da Informação, estudos dos processos documentários com abordagens cognitivas viabilizam o desenvolvimento de pesquisas qualitativas que propiciam uma nova visão do conhecimento de seus profissionais, bem como de suas representações sobre o conhecimento assimilado ou adquirido com vistas á socialização (Dal'Evedove; Fujita, 2008).

Para Frohmann (1990) a Ciência da Informação é uma prática social e sua visão ótica sob a perspectiva cognitiva restringe sua abrangência, por essa não considerar o processo social de construção dos processos informacionais.

No tratamento temático da informação, exemplificado pela catalogação de assunto e pela indexação, a análise e identificação do conteúdo são realizadas por meio da cognição, por isso ela não é um processo sistemático de etapas consecutivas, mas é um processamento mental de informações, que depende de conhecimento prévio e é composta de habilidades operatórias de pensamento (Oliveira, 2005).

Nesse âmbito, a catalogação de assunto e a [...] indexação são colaborativos [...] no delineamento do contexto sociocognitivo do indexador [...] em bibliotecas universitárias, na medida em que essas atividades envolvem os modelos mentais desses sujeitos inseridos em seus ambientes sócioculturais, conforme apresentado nos subitens desta pesquisa. (Boccato, 2009, p. 123).

Diante do exposto e de acordo com o objetivo de nossa pesquisa, foram desenvolvidos os procedimentos metodológicos que se constituíram de estudo diagnóstico composto por duas partes: a) estudo do funcionamento e procedimentos do tratamento de informações documentárias na Rede de Bibliotecas da Universidade Estadual Paulista (UNESP) na perspectiva da Coordenadoria Geral de Bibliotecas (CGB); b) estudo do funcionamento e procedimentos do tratamento de informações na Rede de Bibliotecas da UNESP na perspectiva do catalogador.

Para a realização da primeira parte, foram aplicados questionários de diagnóstico organizacional aos diretores das bibliotecas universitárias. A elaboração do questionário foi fundamentada em Almeida (2005, p. 53-55) para identificar itens organizacionais, materiais, de procedimentos e processos, documentários e de pessoas que constituem o contexto sociocognitivo do catalogador.

A segunda parte, sobre o funcionamento e procedimentos do tratamento de informações na Rede de Bibliotecas da UNESP na perspectiva do catalogador, utilizou a técnica introspectiva do protocolo verbal nas seguintes modalidades:

1. Protocolo verbal individual $(\mathrm{PVI})$ realizado com bibliotecários catalogadores (1) para 
identificação dos procedimentos de análise de assunto na catalogação de livros, bem como dificuldades e restrições;

2. Protocolo verbal em grupo (PVG) com bibliotecários chefes, bibliotecários catalogadores, bibliotecários de referência, usuários docentes pesquisadores, líderes de grupos de pesquisa, e usuários discentes de graduação e pós-graduação.

A rede de Bibliotecas da UNESP é composta por trinta e duas bibliotecas de unidades universitárias e unidades complementares em vinte e três cidades do Estado de São Paulo, Brasil. Os registros de todas as bibliotecas da UNESP estão disponíveis em catálogo central de acesso público via Internet, denominado ATHENA. O sistema ALEPH é o software utilizado para automação dos serviços de aquisição, registro, catalogação, empréstimo e controle de periódicos.

As coletas de dados foram realizadas em uma amostra de nove bibliotecas da UNESP das três áreas do conhecimento - Humanas, Exatas e Biológicas - respectivamente, Pedagogia, Engenharia Civil e Odontologia, cujas transcrições geraram uma grande massa de dados. Considerando a abordagem sociocognitiva na interação do ambiente (biblioteca) e das diferentes perspectivas oriundas dos diversos sujeitos participantes das coletas (bibliotecários chefes, de referência, catalogadores e usuários discentes e docentes) ressaltamos a complexidade que envolve a análise dessas transcrições.

As aplicações dos Protocolos Verbais, na modalidade Individual e em Grupo, foram realizadas nos locais da amostra de nove bibliotecas universitárias com o deslocamento das pesquisadoras e envolvendo a participação efetiva de um total de cinquenta e quatro pessoas nas coletas de dados.

A técnica de coleta de dados introspectivos denominada Protocolo Verbal consiste na gravação da exteriorização verbal do pensamento de um ou mais indivíduos durante a realização de uma tarefa. O "Pensar alto" do sujeito é gravado e transcrito literalmente.

O protocolo verbal em grupo consiste na reunião de sujeitos participantes e pesquisador para a leitura de um texto e discussão de temas suscitados pelo mesmo. Nesse caso, o pesquisador interage como um dos sujeitos participantes com uma única função a mais, controlar o gravador.

Ambos os protocolos verbais seguem procedimentos semelhantes para sua aplicação:

A) Procedimentos anteriores à coleta de dados
- Definição do universo da pesquisa:

PVG e PVI: bibliotecas universitárias da Rede de Bibliotecas UNESP;

- Seleção do Texto-Base:

PVG: trecho entre as páginas 205 e 208 do seguinte artigo: DIAS, Eduardo Wense; NAVES, Madalena Martins Lopes; MOURA, Maria Aparecida. O usuário-pesquisador e a análise de assunto. Perspectivas em Ciência da Informação., Belo Horizonte, v. 6, n. 2, p. 205 - 221, jul./dez. 2001.

$\mathrm{PVI}$ : o próprio registro bibliográfico a ser catalogado para compor o catálogo - base de dados Athena - da Rede de Bibliotecas da UNESP;

- Definição da tarefa:

PVG: discussão do texto-base previamente referenciado

PVI: foi solicitado aos catalogadores que realizassem a catalogação de livros em suas três variações: item considerado original (Catalogação Original - CO), registro aproveitável (RA) e o registro idêntico (Identidade Total IT);

- Seleção dos Sujeitos:

PVG: 3 bibliotecários (chefe, catalogador e de referência), 1 docente e 1 discente.

PVI: população de bibliotecários catalogadores representativa das três áreas do conhecimento - Humanas, Exatas e Biológicas - respectivamente, Pedagogia, Engenharia Civil e Odontologia em nove bibliotecas da UNESP;

- Conversa informal com os sujeitos:

Nesta conversa, as pesquisadoras fizeram contato com os sujeitos por intermédio da Coordenadoria Geral de Bibliotecas explicando os objetivos da pesquisa, a metodologia utilizada e agendando o dia para a coleta de dados. Todos os participantes tiveram suas identidades preservadas.

B) Procedimentos durante a coleta de dados

PVG: após a leitura do texto-base, iniciou-se a discussão, em que o pesquisador fez as intervenções necessárias de modo a instigar os participantes. Toda a discussão foi gravada e transcrita na íntegra.

PVI: toda a exteriorização do pensamento feita pelo catalogador durante a execução da tarefa de catalogação foi gravada com o auxílio de um aparelho de MP3;

C) Procedimentos posteriores à coleta de dados 
- Transcrição das gravações:

PVG: após a gravação da discussão do texto pelos sujeitos, foi feita a transcrição literal com a identificação das fontes das falas individuais. Essa identificação foi feita da seguinte forma: Bibliotecário-Chefe; Bibliotecário de Referência; Bibliotecário Catalogador; Docente; Aluno; Pesquisador;

PVI: após a gravação do "pensar alto" durante a catalogação, foi feita a transcrição literal das gravações das falas dos sujeitos.

- Análise das transcrições:

Com a transcrição pronta, foi feita uma leitura detalhada dos dados em busca de fenômenos significativos e recorrentes para construir categorias de análise que permitissem a análise dos dados de forma organizada e eficiente. Posteriormente à construção das categorias, voltou-se aos dados novamente para retirar trechos da transcrição que exemplifiquem cada categoria.

\section{Resultados}

Os resultados apresentados visam demonstrar a visão do contexto em que a política de indexação está inserida. Para tanto, foram analisados os questionários de diagnóstico organizacional respondidos pelos diretores das bibliotecas universitárias e os protocolos verbais individual e em grupo.

A análise dos questionários dos diagnósticos organizacionais revelou uma estrutura composta das seções de técnicas de referência e atendimento ao usuário e de processamento técnico. Pela análise de quantitativo de pessoal e distribuição de atividades das bibliotecas, observouse que um mesmo bibliotecário realiza tanto a catalogação de forma quanto a de conteúdo.

Os resultados obtidos das análises qualitativas dos dados coletados com a aplicação da técnica do Protocolo Verbal, na modalidade individual e em grupo, foram selecionados e agrupados em duas categorias de análise consideradas importantes para os objetivos do estudo diagnóstico, quais sejam:

\subsection{O processo de indexação na catalogação}

Cada bibliotecário se apoiou em sua formação inicial, na linguagem documentária utilizada e no conhecimento prévio que possui da área de assunto.

Notamos que não há limite de termos para a catalogação de assunto e, dessa maneira, a inexistência, também, de manual específico para indexação.

Exemplo: PVG, Engenharia Civil,

13 Pesquisadora

Existe algum limite para determinação do assunto?

14 Bibliotecário Catalogador

Não, eu não coloco esse limite. Enquanto eu for achando, eu vou colocando. Mas eu acho que mais ou menos seis termos já está suficiente. Enquanto eu consigo, o termo normalizado por exemplo, eu vou colocando. Ou então, quando não dá, eu já saio do normalizado e vou colocando. Eu nunca ouvi falar nada sobre isso. Eu acho que às vezes, o catalogador coloca um limite para ficar um trabalho mais ágil, porque é um serviço demorado, é a parte mais demorada. Quantos assuntos o documento abordar, pode colocar lá.

A análise de assunto não é realizada adequadamente comprometendo a representação e o uso da linguagem com cabeçalhos de assuntos não correspondentes ao nível em que o assunto é efetivamente abordado pelo autor, verificado: pela falta de sistematização na realização da leitura documentária pelos bibliotecários das três áreas do conhecimento; pela utilização da ficha catalográfica e pelos sistemas de classificação como fontes de informações para identificar e selecionar os assuntos; e pela realização da indexação classificatória.

Exemplo: PVI - Pedagogia

Bibliotecário Catalogador

Então agora vamos para o campo de assunto. A outra bibliotecária já trouxe a ficha para mim.. Somente "Educação" a gente já sabe que é autorizado, especialmente para dar firmeza, vamos lá o assunto. [...] Aí vou para o sumário, "Crianças na prisão", às vezes têm pessoas que pesquisam. Vamos ver, não temos criança na prisão a gente tem FEBEM, também não tem por FEBEM, aí eu dou uma olhadinha no prefácio. Realmente deixaram um só assunto na ficha catalográfica em papel porque é complicado colocar assunto e fala sobre criança. Então eu acho que tem que ser "Educação" e "Criança" porque só "Educação" ficou estranho, porque já que está falando aqui que é educação de crianças e crianças na prisão, então eu posso colocar "Educação" e "Criança". Vou deixar só educação como foi autorizado a "Educação da criança.

Além disso, observamos a necessidade de realização da indexação analítica de documentos exemplificada por capítulos de livros - e a questão dos procedimentos de análise e representação da informação feitos pelos bibliotecários terem como objetivo atender a demanda usuária.

Exemplo: PVG, Odontologia,

25 Bibliotecário Catalogador 
Então, quando a gente faz o trabalho de levantamento junto com o usuário, é uma coisa muito apta até para fazer a catalogação e faz a catalogação geral do material, se a gente tivesse um tempo maior, pegaríamos esse material e faríamos uma indexação de capítulo por capítulo do livro, e jogar isso em um índice para o usuário recuperar.

4.2. A mediação da linguagem documentária na representação e recuperação da informação

A utilização da linguagem documentária para a representação da informação limita-se à confirmação e validação dos cabeçalhos.

As principais preocupações e sugestões dos catalogadores, em relação à linguagem que utilizam - Lista de Cabeçalhos de Assunto da Rede Bibliodata (LCARB) - linguagem documentária utilizada para a representação e recuperação da informação no catálogo coletivo online ATHENA -, referem-se à atualização, especificidade e compatibilidade.

\section{Exemplo: PVG - Engenharia Civil \\ 3 Bibliotecário Catalogador}

Eu faço assim, pode não ser a melhor forma. Eu pego o material e coloco de forma geral "Engenharia Civil" ai eu vou na ficha catalográfica, na orelha do livro, na introdução do autor e o que não dá para se encaixar no cabeçalho controlado eu coloco no assunto local. Nós temos um campo que não precisa estar autorizado pelo Bibliodata que a gente consulta, né. E aí eu fico assim um pouco mais tranqüila porque eu tenho a impressão que isso ajuda um pouco Às vezes, a gente tenta pedir para inserir um assunto.

Nesse contexto, ressaltamos que a LCARB não possui vocabulário especializado, atualizado e estruturas sintático-semântica consistentes ocasionadas por: presença de termos ambíguos, falta de remissivas, problemas entre cabeçalhos no singular e plural e linguagem de busca do usuário inadequada e insuficiente.

Para tanto, observamos a adoção de outros sistemas de organização do conhecimento para os cabeçalhos não disponíveis na LCARB, entre eles citamos o DeCS - Descritores em Ciências da Saúde da BIREME - Centro Latinoamericano e do Caribe de Informação em Ciências da Saúde.

A opção dos bibliotecários catalogadores, por representar os conceitos por termos genéricos foi uma constante, o que ocasionou resultados muito abrangentes na busca por assunto.

Outras manifestações foram realizadas por eles a saber: disponibilidade da linguagem na interface de entrada de dados (catalogação) do catálogo online para a representação da informação a partir da própria linguagem em conexão com o formato bibliográfico utilizado; o reconhecimento da importância do se conhecer a área científica a ser representada pela linguagem; o catálogo deve atuar a semelhança de uma base de dados, a necessidade da linguagem documentária ser disponibilizada para consulta e uso pelo usuário na busca por assunto no sistema; e sobre a necessidade de construção de um vocabulário controlado para a Rede UNESP.

\section{Considerações finais}

A indexação na catalogação é reconhecida como uma prática intelectual do bibliotecário em sistemas de recuperação da informação que, inserido em seu contexto social, realiza essa atividade visando os objetivos organizacionais e as necessidades de representação e recuperação da informação científica especializada. Em vista disso, observamos que a perspectiva sociocognitiva torna-se um elemento fundamental nesse processo, como abordagem metodológi$\mathrm{ca}$, em pesquisa qualitativa com o uso da técnica do protocolo verbal como instrumento introspectivo de coleta de dados.

Essa metodologia tem como ponto focal o sujeito que realiza uma determinada atividade e sua cognição em relação ao seu contexto de produção. O foco está no contexto de tratamento temático do bibliotecário catalogador constituído de objetivos da indexação, política de indexação, regras e procedimentos do manual de indexação, a linguagem para representação e mediação visando os interesses de busca do usuário. Esse cenário demonstra o contexto em que esse bibliotecário está inserido e que certamente o influenciará durante o processamento textual para os objetivos de indexação.

Nesse sentido, esta pesquisa possibilitou-nos verificar o desempenho do bibliotecário catalogador no seu "fazer" diário na indexação, a compreensão que possuíam sobre esse processo e sobre o uso da linguagem documentária adotada pelo sistema a partir de uma visão interacionista entre o meio e a percepção dos indivíduos envolvidos no desenvolvimento dessa atividade.

Concluímos, portanto, que é tendência dos catálogos atuarem como bases de dados e, dessa forma, o bibliotecário catalogador precisa ter consciência da importância de sua atuação na representação de assuntos, apresentando uma postura compromissada semelhante à de um indexador que trabalha na produção dessas bases. 


\section{Notas}

(1) No contexto da Rede de Bibliotecas da UNESP, os bibliotecários indexadores correspondem aos bibliotecários catalogadores que desenvolvem as atividades de catalogação e indexação.

\section{Referências bibliográficas}

Almeida, M. C. B. de. (2005). Planejamento de bibliotecas e serviços de informação. 2. ed. rev.e ampl. Brasília: Briquet de Lemos, 2005. 4, 53-92. ISBN 85-8563-727-7.

Boccato, V. R. C. (2009). Avaliação do uso de linguagem documentária em catálogos coletivos de bibliotecas universitárias: um estudo sociocognitivo com protocolo verbal. Marília: Universidade Estadual Paulista, Faculdade de Filosofia e Ciências, 2009. Tese de Doutorado. http:// www.unesp.br/cgb/int_conteudo_sem_img.php?conteud $o=562 \cdot(2010-03-13)$

Cutter, C. A. (1904). Rules for a dictionary catalog. $4^{\text {th }}$ ed. rewritten. Washington: Gov't Printing Office, 1904.

Dal'evedove, p. R.; Fujita, M. S. L. A Cognição profissional de catalogadores de assunto em contexto de biblioteca universitária. // Seminário Nacional de Bibliotecas Universitárias- SNBU, 15., 2008, São Paulo. Anais... São Paulo: CRUESP, 2008. p. 1-15. http://www.sbu.unicamp. br/snbu2008/anais/site/pdfs/3198.pdf. (2010-03-23).

Fiúza, M. M. (1985). da Catalogação de assunto. // Revista da Escola de Biblioteconomia da UFMG. ISSN. 01000829. 14:2 (set. 1985) 257-269.

Frohmann, B. (1990). Rules of indexing: a critique of mentalism in information retrieval theory. // Journal of Documentation. ISSN 0022-0418. 46:2 (1990) 81-101.

Guimarães, J. A. C. (2003). A análise documentária no âmbito do tratamento da informação: elementos históricos e conceituais. // Rodrigues, G. M; Lopes, I. L. (orgs.). Organização e representação do conhecimento na perspectiva da Ciência da Informação. Brasília: Thesaurus, 2003. ISBN 85-7062-399-2. 100-117. (Estudos avançados em Ciência da Informação; 2).

Lancaster, F. W. (2004). Indexação e resumos: teoria e prática. Tradução de Antonio Agenor Briquet de Lemos. 2. ed. Brasília: Briquet de Lemos, 2004. ISBN 85$8563724=2$

Neves, D. A. (2006). Ciência da informação e cognição humana: uma abordagem do processamento da informação. // Ciência da Informação. ISSN 1518-8353. 35:1 (jan./abr. 2006) 39-44.

Oliveira, D. S. de. (2005). Estratégias para o ensino de indexação: o uso do protocolo verbal interativo como recurso pedagógico de indexadores aprendizes. Marília: Universidade Estadual Paulista, Faculdade de Filosofia e Ciências, 2005. Monografia de Graduação.

Pinto Molina, M. (1993). Análisis documental: fundamentos y procedimientos. 2. ed. rev. y aum. Madrid: EUDEMA, 1993. ISBN 84-7754-070-5.

Rubi, M. P. (2008). Política de indexação para construção de catálogos coletivos em bibliotecas universitárias. Marília: Faculdade de Filosofia e Ciências, Universidade Estadual Paulista, 2008. Tese de Doutorado http://www.unesp.br/cgb/int_conteudo_sem_img.php?co nteudo=562 . (2010-03-07)

Silva, M. dos R. da; Fujita, M. S. L. (2004). A prática de indexação: análise evolutiva de tendências teóricas e metodológicas. // Transinformação. ISSN 0103-3786. 16:2 (2004) 133-161.

Tillett, B. (ed.). (2009). IFLA cataloguing principles: statement of international cataloguing principles (ICP) and its glossary. München: K. G. Saur Verlag, 2009. ISB 978-
3598-24285-4. (Series on Bibliographic Control, v. 37). http://www.ifla.org/VII/s13/icc/imeicc-statement_of_principles-2008.pdf. (2010-03-30).

UNISIST. (1981). Princípios de indexação. // Revista da Escola de Biblioteconomia da UFMG. ISSN 0100-0829. 10:1 (mar. 1981) 83-94.

Van Slype, G. (1991). Los lenguajes de indización: concepción, construcción y utilización en los sistemas documentales. Traducción del frances: Pedro Hípola, Félix de Moya. Madrid: Fundación Germán Sanchez Ruipérez, 1991. ISBN 8436805844.

Recibido: 2010-04-15. Revisado: 2010-06-22. Aceptado: 2007-07-06. 